\section{Zinc supplementation in children with asthma exacerbation}

\section{Sanguansak Rerksuppaphol, 1 Lakkana Rerksuppaphol2 \\ 1Department of Pediatrics and 2Department of Preventive Medicine, Faculty of Medicine, Srinakharinwirot University, Thailand}

\begin{abstract}
Zinc deficiency has demonstrated an association with the risk of asthma. This study aimed to evaluate the efficacy of zinc supplementation in reducing the severity of childhood asthma exacerbation. A number of 42 children with asthma exacerbation admitted to the hospital were randomized to receive either zinc bis-glycinate (30 mg elemental zinc/day) or a placebo in adjuvant to the standard treatment. The pediatric respiratory assessment measure (PRAM) was used to measure the asthma severity. The primary outcome was a change in asthma severity from the baseline to the end of study. The study found that PRAM score in the zinc group showed a more rapid decrease compared to the control group at the 24-hour $(2.2 \pm 1.3$ vs. $1.2 \pm 1.3 ; \mathrm{P}=0.015)$ and 48 -hour $(3.4 \pm 2.0$ vs. $2.2 \pm 1.8 ; \mathrm{P}=0.042)$ intervals. At admission, overall mean serum zinc level was $63.8 \mu \mathrm{g} / \mathrm{dL}$ and $57.1 \%$ of children had zinc deficiency with no difference in prevalence between groups. PRAM scores did not differ between children with low and normal zinc status. In conclusion, zinc supplementation as the adjuvant therapy to the standard treatment during asthma exacerbation resulted in rapid lessening of severity.
\end{abstract}

\section{Introduction}

Asthma is the most common chronic disorder of the airways in children, affecting approximately $10 \%$ of children worldwide. ${ }^{1}$ It is responsible for varied economic burdens including direct costs through consumption of resources (e.g., hospitalizations, physician visits, and medications), as well as indirect costs through loss of productivity.

Asthma is a complex disease, the pathophysiology and pathogenesis of which are still not fully understood. Airway inflammation is considered as one of the pathogenesis and pathophysiology of asthma. ${ }^{2}$ The inflammatory processes in the airways cause the limitation of airflow and result in the clinical manifesta- tions of asthma such as recurrent episodes of coughing, wheezing, breathlessness, chest tightness and shortness of breath. ${ }^{3} \mathrm{~A}$ variety of inflammatory cells including mast cells, eosinophils, lymphocytes and neutrophils infiltrate the airway epithelium and release many pro-inflammatory mediators, leading to inflammatory responses, bronchospasm, airway edema, acute and persistent inflammation and airway remodeling. ${ }^{4}$ The activation of immune cells leads to the release of several stored toxic mediators, including free radicals and reactive oxygen species. 5 The increase in oxidative stress in asthma is associated with the declination of antioxidants which contributes to oxidative damage to mitochondrial respiratory chain complexes, leading to airway inflammation, vascular permeability, mucus hypersecretion, smooth muscle contraction and epithelial shedding. ${ }^{6,7}$ Since oxidative stress is involved in the propagation of the inflammatory response in asthma, the supplementation of antioxidants has been proposed as one strategy to combat this disorder.

Zinc is an essential trace element for human metabolism, which contributes to enzyme activities, protein structuring and the regulation of gene expression. It also plays an important role in the modulation of the immune system as well as acting as an antioxidant, anti-inflammatory and anti-apoptosis. ${ }^{8-}$ 11 Several studies observed the association between low zinc statuses either in serum, hair or sputum and asthma prevalence. ${ }^{12-17} \mathrm{~A}$ small number of studies have been conducted to evaluate the efficacy of zinc supplementation for improvement lung functions or clinical symptoms of asthma during non-exacerbation; however, the results were not consistent.18-20 Up to present, there is no report of zinc supplementation improving the clinical severity during asthmatic exacerbation. The aim of the present study was to evaluate the efficacy of the zinc amino acid chelate in reducing the severity of asthma in children admitted to the hospital due to asthma exacerbation.

\section{Materials and Methods}

\section{Study design and subjects}

A double-blinded randomized control trial was conducted between January and December 2015 in children aged 2-16 years admitted to the pediatric unit of Srinakharinwirot University Hospital with a diagnosis of acute asthma exacerbation. Decisions regarding admission and general management of asthma were made, respectively, by the emergency and attending physicians.

The children who were eligible to partici-
Correspondence: Sanguansak Rerksuppaphol, Department of Pediatrics, Faculty of Medicine, Srinakhariwirot University, 62 Mo 7, RangsitNakorn Nayok Rd., NakornNayok 26120, Thailand Tel.: +66.817231766 - Fax: +66.37395275.

E-mail: sanguansak_r@hotmail.com

Key words: Asthma; Child; Dietary supplement; Zinc.

Acknowledgements: the authors would thank all children and their parents who participated in the study and the many people that assisted with this research project.

Contributions: the authors contributed equally.

Conflict of interest: the authors declare no potential conflict of interest.

Funding: this study was supported by grants from Srinakharinwirot University, Thailand.

Received for publication: 8 July 2016 . Accepted for publication: 31 August 2016.

This work is licensed under a Creative Commons Attribution NonCommercial 4.0 License (CC BYNC 4.0).

(C) Copyright S. Rerksuppaphol and

L. Rerksuppaphol, 2016

Licensee PAGEPress, Italy

Pediatric Reports 2016; 8:6685

doi:10.4081/pr.2016.6685

pate in the study were only those who had asthma exacerbation as well as a background of asthma defined as either: i) at least one previous episode of $\beta_{2}$-agonist-responsive wheeze in a child two years of age or over; or ii) a prior diagnosis of asthma, made by a pediatrician, or clinician of comparable experience. An asthma exacerbation was defined as those patients who had one or all of the following clinical features:21 dyspnea, wheeze, acute cough, increase work of breathing, increased requirement for $\beta_{2}$-agonist from baseline use, and oxygen saturation $<95 \%$.

Excluded from the study were those patients who: i) had underlying diseases such as bronchopulmonary dysplasia, congenital heart disease, renal disease, neurological disease, immune deficiency and malignancy; ii) already taking vitamin or mineral supplements regularly or having allergy to them; or iii) could not tolerate oral feeding.

The study was done according to the principles of the Declaration of Helsinki. The protocol was approved by the ethics committee of the Srinakharinwirot University. Informed written consent and assent were obtained from parents or legal guardians and children, respectively, before enrolment. Parents and 
children were allowed to withdraw from the study at any time and the reason for withdrawal was recorded. The zinc supplements and placebo were purchased from the manufacturing company. The company had not contributed to the design of the study, the accrual or analysis of the data, or in any processes related to the study in any way. This study is registered with the Thai Clinical Trials Registry (TCTR20141212001).

\section{Intervention}

After enrolment, patients were randomized to receive zinc supplementation or a placebo. Randomization was done by a statistician who was not involved in the implementation phase of the study. The investigators, attending clinicians, children and parents were masked to the intervention. The code of randomization sequence was opened only after the study was completed.

Participants in the zinc supplementation group were given chelated zinc in the form of zinc bis-glycinate (15 mg elemental zinc) twice a day until their discharge from the hospital and up to a maximum of 4 days. This was supplied in powder form in single dose sachets (Qualimed ${ }^{\circledR}$, Bangkok, Thailand). The contents of the sachet were dissolved in a glass of water before administration. Participants in the control group were given an ORS-based placebo, which was prepared by the same manufacturer and was in an identical package, and had identical color and taste. Treatment of asthma, observation and discharge decision about the patients was done by their attending physicians who were not involved in the implementation phase of the study.

\section{Data collection and monitoring}

Baseline, demographic characteristics and clinical data were recorded by trained research staff. Weight was measured to the nearest 100 g using an electronic scale and height was measured to the nearest millimeter. Body mass index (BMI) was calculated as the ratio of weight/(height) ${ }^{2}\left(\mathrm{~kg} / \mathrm{m}^{2}\right)$. The severity of asthma exacerbation was assessed using the pediatric respiratory assessment measure (PRAM) score at baseline and again at 24-hour intervals until the end of study by the same staff. 22 Serum zinc levels were measured by flame atomic absorption spectrometry at baseline and at 4 days after supplementation (or before discharged from hospital). The time of the blood drawing and fasting status of patients were recorded. Patients who had serum zinc level less than the lower cutoffs regards to age, sex, fasting status and time of day of blood collection were defined as having zinc deficiency. In summary, the lower cutoffs for serum zinc concentrations were: i) age $<10$ years: morning non-fasting $65 \mu \mathrm{g} / \mathrm{dL}$, afternoon $57 \mu \mathrm{g} / \mathrm{dL}$; ii) females, age $\geq 10$ years: morning fasting 70 $\mu \mathrm{g} / \mathrm{dL}$, morning non-fasting $66 \mu \mathrm{g} / \mathrm{dL}$, afternoon $59 \mu \mathrm{g} / \mathrm{dL}$; and iii) males, age $\geq 10$ years: morning fasting $74 \mu \mathrm{g} / \mathrm{dL}$, morning non-fasting $70 \mu \mathrm{g} / \mathrm{dL}$, afternoon $61 \mu \mathrm{g} / \mathrm{dL} .23$ Openended questions were asked to assess side effects in both groups. Compliance to treatment was assessed by the sum of drug intakes.

\section{Outcome measurement}

The primary outcome was change in severity of asthma assessed by PRAM score from the baseline to the end of the study. The secondary outcomes were change in serum zinc levels from the baseline to the end of study and prevalence of zinc deficiency.

\section{Statistical analysis}

The results were descriptively presented as mean and standard deviation, median and interquartile range, or frequency and percentage. The Pearson chi-square or the Fisher exact test were used to compare proportion between the groups. The normality of distribution was checked by Kolmogorov-Smirnov test. Normally distributed continuous data were compared using a student t-test whereas notnormally distributed data were compared by Mann-Whitney U-test. The change in serum zinc levels from the baseline was assessed by paired t-test and presented as means and 95\% confidence interval (CI). A P-value less than 0.05 was considered statistically significant.

\section{Results}

Forty-three children with acute asthma exacerbation were approached to participate in this study. One subject declined to participate; therefore, a total of 42 patients were allocated to two study groups (21 patients each in the zinc group and the control group). All patients $(26,61.9 \%$ boys) completed the assigned treatment and were included in the final analysis. Baseline demographic and clinical characteristics are shown in Table 1 . The mean age, weight and BMI of the study group were 6.5 years (range, 2.0-16.0 years), $26.2 \mathrm{~kg}$ (range, $9.6-52.9 \mathrm{~kg}$ ) and $17.75 \mathrm{~kg} / \mathrm{m}^{2}$ (range, $10.90-32.74 \mathrm{~kg} / \mathrm{m}^{2}$ ), respectively. The frequency of clinical presentation was as follows: acute cough in 40 patients (95.2\%), wheezing in 39 patients (92.9\%), increased work of breathing in 37 patients (88.1\%) and dyspnea in 32 patients $(76.2 \%)$. There were no significant differences in demographic and clinical characteristics between groups.

At admission, the mean PRAM score of the zinc supplementation group was comparable with those of the placebo group (5.4 \pm 2.2 and $4.5 \pm 1.9$, respectively; $\mathrm{P}=0.181$ ). There were no significant differences in receiving initial medical treatment for asthma exacerbation between groups. All patients in both groups received the initial treatment of asthma exacerbation by inhaled salbutamol; moreover, 15 and 14 patients in the zinc supplementation and placebo groups, respectively, had an addon therapy of inhaled ipratropium bromide $(\mathrm{P}=1.000)$. Thirteen patients in the zinc group and 17 patients in the control group received systemic steroids as part of their treatment $(\mathrm{P}=0.306)$.

The PRAM scores of both groups decreased subsequently during treatment (Table 2). There were no significant differences between groups in PRAM scores at 24, 48, 72 and 96 hours after treatment; however, patients in the zinc supplementation group had significant

Table 1. Demographic and clinical characteristics (values are mean \pm standard deviation).

\begin{tabular}{lccc} 
& $\begin{array}{c}\text { Treatment } \\
(\mathrm{n}=21)\end{array}$ & $\begin{array}{c}\text { Placebo } \\
(\mathrm{n}=21)\end{array}$ & P-value \\
Boys, $\mathrm{n}(\%)$ & $16(76.2)$ & $10(47.6)$ & 0.111 \\
Age, $\mathrm{yr}$ & $5.9 \pm 2.7$ & $7.2 \pm 3.3$ & 0.152 \\
\hline Weight, median $\mathrm{kg}(\mathrm{IQR})$ & $21.0(14.3-28.2)$ & $26.9(19.4-39.7)$ & 0.054 \\
Body mass index, $\mathrm{kg} / \mathrm{m}^{2}$ & $16.55 \pm 4.67$ & $18.91 \pm 4.29$ & 0.102 \\
\hline Clinical features & & & \\
Dyspnea, $\mathrm{n}(\%)$ & $15(71.4)$ & $17(81.0)$ & 0.719 \\
Wheezing, $\mathrm{n}(\%)$ & $19(90.5)$ & $20(95.2)$ & 1.000 \\
Acute cough, $\mathrm{n}(\%)$ & $20(95.2)$ & $20(95.2)$ & 1.000 \\
Increase of work of breathing, $\mathrm{n}(\%)$ & $19(90.5)$ & $18(85.7)$ & 1.000 \\
Increased requirement for bronchodilator, $\mathrm{n}(\%)$ & $5(23.8)$ & $6(28.6)$ & 1.000 \\
$\mathrm{O}_{2}$ saturation, \% & $96.3 \pm 2.8$ & $96.2 \pm 2.5$ & 0.862 \\
$\mathrm{O}_{2}$ saturation $<95 \%, \mathrm{n}(\%)$ & $4(19.0)$ & $5(23.8)$ & 1.000 \\
Body temperature, ${ }^{\circ} \mathrm{C}$ & $37.5 \pm 0.8$ & $37.5 \pm 0.7$ & 0.888 \\
Respiratory rate, per minute & $32 \pm 9$ & $32 \pm 6$ & 0.967 \\
\hline
\end{tabular}


higher reduction of PRAM scores from their baseline compared to the control group at 24 hours $(2.2 \pm 1.3$ vs. $1.2 \pm 1.3$, respectively; $\mathrm{P}=0.015)$ and at 48 hours $(3.4 \pm 2.0$ vs. $2.2 \pm 1.8$, respectively; $\mathrm{P}=0.042$ ).

The mean serum zinc level upon admission of the study population was $63.8 \pm 17.4 \mu \mathrm{g} / \mathrm{dL}$. There was no significant difference in serum zinc level between the two groups (Table 3). Overall prevalence of zinc deficiency in patients with asthma exacerbation at admission was $57.1 \%$ (24 of 42 patients). The prevalence of zinc deficiency at admission was comparable between groups. Patients who had zinc deficiency at admission had higher PRAM severity scores than in patients who had normal zinc; however, the difference did not reach statistically significance levels $(5.3 \pm 2.1 \mathrm{vs}$. $4.4 \pm 1.9$, respectively; $\mathrm{P}=0.169$ ). At the end of the study, the mean serum zinc levels of the study population was $87.1 \pm 33.6 \mu \mathrm{g} / \mathrm{dL}$. Serum zinc levels were significant increased from their baseline in the zinc supplementation group by $32.3 \mu \mathrm{g} / \mathrm{dL}(95 \%$ CI 14.2 to $50.5 \mu \mathrm{g} / \mathrm{dL}$; $\mathrm{P}=0.001$ ) and the control group by $14.2 \mu \mathrm{g} / \mathrm{dL}$ (95\% CI 5.2 to $23.2 \mu \mathrm{g} / \mathrm{dL} ; \mathrm{P}=0.001$ ); however, there was no significant difference in the rising levels between groups. At the end of the study, 8 (19.0\%) patients still had low serum zinc levels. Patients in the zinc supplementa- tion group had a lower rate of zinc deficiency than in the control group but the difference did not reach statistical significance (9.5 vs. $28.6 \%$, respectively; $\mathrm{P}=0.238$ ).

Average hospital stay was comparable between groups $(3.9 \pm 1.3$ days in the zinc supplementation group vs. $4.1 \pm 1.0$ days in the control group; $\mathrm{P}=0.504)$. No major adverse events were observed in any patient. One patient in the zinc group reported mild nausea, which subsided uneventfully after a day without specific treatment. Compliance was good and similar in both groups; $92.3 \%$ of assigned medications were consumed in both groups.

\section{Discussion}

The present study demonstrates that chelated zinc supplementation (15 mg elemental zinc) twice a day administered to children admitted to the hospital with asthma exacerbation significantly and rapidly decreased the severity of the asthma in the first 48 hours after admission. Approximately half (57\%) of the children with acute asthma exacerbation had zinc deficiency. The majority of the patients who received zinc supplementation (90.5\%) had normal zinc status at the end of

Table 2. Pediatric respiratory assessment measure severity scores during study.

\begin{tabular}{lccc} 
& Treatment & $\begin{array}{c}\text { Placebo } \\
(\mathbf{n}=21)\end{array}$ & $\begin{array}{c}\text { P-value } \\
(\mathbf{n}=21)\end{array}$ \\
Admission & $5.4 \pm 2.2$ & $4.5 \pm 1.9$ & 0.181 \\
24 hours & $3.1 \pm 1.9$ & $3.3 \pm 1.8$ & 0.741 \\
\hline 48 hours & $3.2 \pm 1.2$ & $2.3 \pm 1.8$ & 0.429 \\
72 hours & $1.0 \pm 1.0$ & $1.3 \pm 1.8$ & 0.613 \\
\hline 96 hours & $1.0 \pm 0.9$ & $1.3 \pm 2.7$ & 0.656 \\
& Reduction of PRAM scores from baseline & \\
At 24 hours & $2.2 \pm 1.3$ & $1.2 \pm 1.3$ & 0.015 \\
At 48 hours & $3.4 \pm 2.0$ & $2.2 \pm 1.8$ & 0.042 \\
\hline At 72 hours & $4.4 \pm 2.2^{*}$ & $3.2 \pm 2.0$ & 0.090 \\
At 96 hours & $3.9 \pm 1.7^{\circ}$ & $3.6 \pm 2.3^{\#}$ & 0.693 \\
\hline
\end{tabular}

${ }^{*} \mathrm{n}=18 ;{ }^{\circ} \mathrm{n}=15 ;{ }^{*} \mathrm{n}=14$. PRAM, pediatric respiratory assessment measure.

Table 3. Serum zinc levels of the children with acute asthma exacerbation in the study.

\begin{tabular}{lccc} 
& $\begin{array}{c}\text { Treatment } \\
(\mathrm{n}=21)\end{array}$ & $\begin{array}{c}\text { Placebo } \\
(\mathrm{n}=21)\end{array}$ & P-value \\
Zinc levels at admission, $\mu \mathrm{g} / \mathrm{dL}$ & $62.9 \pm 13.7$ & $64.8 \pm 20.7$ & 0.734 \\
Zinc deficiency, $\mathrm{n}(\%)$ & $12(57.1)$ & $12(57.1)$ & 1.000 \\
\hline Normal levels, $\mathrm{n}(\%)$ & $9(42.9)$ & $9(42.9)$ & \\
Zinc levels at the end, $\mu \mathrm{g} / \mathrm{dL}$, median $(\mathrm{IQR})^{\circ}$ & $84.0(70.0-97.5)$ & $78.0(61.5-95.0)$ & 0.291 \\
\hline Zinc deficiency, $\mathrm{n}(\%)$ & $2(9.5)$ & $6(28.6)$ & 0.238 \\
Normal levels, $\mathrm{n}(\%)$ & $19(90.5)$ & $15(71.4)$ &
\end{tabular}

Values are mean \pm standard deviation or $\mathrm{n}(\%) .{ }^{\circ} \mathrm{IQR}$, Interquartile range.

study. However, the study could not demonstrate statistically significant differences in serum zinc levels at the end of study between the zinc supplementation and non-supplementation groups.

The present finding of the low zinc levels in asthmatic patients is in agreement with previous studies.24 El-Kholy and colleagues found that the mean serum zinc levels in children aged 2-12 years suffering with bronchial asthma was $70.3 \mu \mathrm{g} / \mathrm{dL}$, which was lower than the levels in the healthy controls. ${ }^{24}$ Later study by Khanbabaee and colleagues also found that the mean serum zinc level in pediatric asthma patients was $70.5 \mu \mathrm{g} / \mathrm{dL}$, and that $42 \%$ of patients were hypozincemic. 17 They found that there was a significant association between zinc level and severity of disease. Both studies were conducted to evaluate zinc status, using a cross-sectional study design, during non-exacerbation periods. In contrast, our finding of low serum zinc levels was observed during acute asthma exacerbation. The difference in criteria using for diagnosis of hypozincemia may contribute to the difference in prevalence between studies. Rather than the fixed cutoff levels for diagnosis as in other studies, the present study interpreted the results of the analysis based on the suggested reference data for age, sex, time of day, and time since last meal, as suggested by the International Zinc Nutrition Consultation Group. ${ }^{25}$ Along with the follow-up, zinc levels returned to normal at the recovery phase, even in the non-supplementation group. This is consistent with the previous study and suggests that zinc has a relationship with severity of asthma.

There are few studies on the benefits of zinc supplementation in asthma or airway hyperresponsiveness. ${ }^{18,19,26}$ Morgan and colleagues reported benefits of zinc supplementation given prior to allergen exposure on decreased airway hyperresponsiveness and serum $\operatorname{IgE}$ levels in an animal model. ${ }^{26}$ Bitagi and colleagues reported the benefit of zinc supplementation on pulmonary functions and sputum inflammatory markers in children with moderate persistent asthma.18 Ghaffari and colleagues found that the $50 \mathrm{mg}$ zinc supplement in children with asthma attending at outpatient clinic yielded significant improvements in clinical symptoms such as cough, wheezing, dyspnea and pulmonary functions during follow-up. ${ }^{19}$ However, Pouramjad and colleagues reported the conflicting results that zinc supplementation by dosage of $50 \mathrm{mg}$ on alternate days did not have any significant beneficial effect on pulmonary function parameters. ${ }^{20}$ The present study used zinc supplementation on a daily basis but given in lower dosage (30 mg of zinc/day) compared to Ghaffari study. ${ }^{19}$ However, we found relatively the same effectiveness in improving clinical symptoms of asthma as in the previous study. 
The dosage of zinc in this study was approximately 3-4 times of the recommended daily allowances as used for treatment of zinc deficiency. ${ }^{27}$ As zinc is essential for multiple aspects of metabolism and its daily requirement basis, this may explain the negative results of alternate-day supplementation employed by the Pouramjad study. ${ }^{20}$ A chelated form of zinc, such as the one used in the present study, is better absorbed than inorganic forms, ensuring virtually no adverse effects, very good compliance and effective blinding and may have better systemic effects. The present study used PRAM scores for assessment of asthma severity and response to treatment. PRAM score, an objective assessment for asthma severity, is recognized for its appropriateness in this context, ability to distinguish across severity levels, good internal consistency, and inter-reliability for all pediatric age groups. ${ }^{22}$ During acute asthma exacerbation, it is nearly impossible to assess lung function tests in young children by other methods such as peak expiratory flow rate or spirometry because of poor coordination, severity of illness and poor familiarity with the technique. ${ }^{22}$ Even though the present study found a significant and rapid improvement in PRAM score in the zinc supplementation in the first 48 hours after admission, duration of admission and severity score at the end were not significantly different between groups. These findings may be explained by how patients in both groups received the same medical treatment for asthma exacerbation as per the standard recommendation for asthma management including short acting $\beta 2$-agonists, an inhaled anticholinergic and systemic corticosteroids. ${ }^{28}$

The exact mechanisms of zinc in rapidly reducing asthma severity are not clear, the possible reasons include its usefulness as an antioxidant, an organelle stabilizer, anti-apoptotic agent and anti-inflammatory, involving $\mathrm{T}$ cell cytokine expression, NF kappaB signaling PPAR, COX-2 expression and down-regulation iNOS. ${ }^{9-11}$ Zinc is essential and plays many maintenance roles in cellular metabolism and integrity, including airway epithelium. Mild zinc deficiency has been reported to complicate worsened airway inflammation and lung functions. ${ }^{29}$ Zinc deficiency is common in Thailand with an estimated prevalence of more than $40 \% .25$ The finding of high prevalence of zinc deficiency in children with asthma exacerbation, and how zinc levels returned to normal in the recovery phase in the present study, may suggest the possibility of further studies on the benefits of the long-term zinc supplementation for preventing asthma exacerbation, especially in children with marginal zinc deficiency like those found in Thailand.

The present study had some limitations. The small sample size might not be sufficiently large to detect modest benefits. However, the present study had detected some benefits of faster reduction of severity of asthma exacerbation. This study did not evaluate dietary intake of zinc. We assumed that the dietary intake of zinc was similar between the two groups, matched by randomization controlled masked design. We measured the benefit outcomes only until the end of supplementation. We do not know whether the benefits would have been sustained with a longer duration of supplementation, or whether this would continue to provide protecting effects on asthma exacerbation. Long-term prospective studies are needed to evaluate the maximum benefits against childhood asthma. We did not investigate whether the beneficial effects on reduction of severity of asthma exacerbation was from either the pharmacological effect of zinc supplementation or the mixed effects of zinc and medical treatment for asthma. For ethical reasons, it was impossible to conduct the study without the prescribed standard recommendation of medical treatment in children during asthma exacerbation. We believe that both treatments have synergistic effects, as zinc helps to decrease airway inflammation, which is one of the physiological characteristics of asthma. ${ }^{3}$

\section{Conclusions}

The present study shows the zinc supplementation (30 mg elemental zinc/day), as the adjuvant therapy to the standard medical treatment, results in rapid lessening of severity during asthma exacerbation. However, the study could not demonstrate significant difference in the duration of admission. There was no significant adverse event with this treatment.

\section{References}

1. Asher MI, Montefort S, Bjorksten B, et al. Worldwide time trends in the prevalence of symptoms of asthma, allergic rhinoconjunctivitis, and eczema in childhood: ISAAC Phases One and Three repeat multicountry cross-sectional surveys. Lancet 2006;368:733-43.

2. Holgate ST. Pathogenesis of asthma. Clin Exp Allergy 2008;38:872-97.

3. Hedlin G, Konradsen J, Bush A. An update on paediatric asthma. Eur Respir Rev 2012;21:175-85.

4. Barnes PJ. The cytokine network in asthma and chronic obstructive pulmonary disease. J Clin Invest 2008;118:3546-56.

5. Kirkham P, Rahman I. Oxidative stress in asthma and COPD: antioxidants as a ther- apeutic strategy. Pharmacol Ther 2006;111:476-94.

6. Aguilera-Aguirre L, Bacsi A, SaavedraMolina A, et al. Mitochondrial dysfunction increases allergic airway inflammation. J Immunol 2009;183:5379-87.

7. Nadeem A, Masood A, Siddiqui N. Oxidantantioxidant imbalance in asthma: scientific evidence, epidemiological data and possible therapeutic options. Ther Adv Respir Dis 2008;2:215-35.

8. Shankar AH, Prasad AS. Zinc and immune function: the biological basis of altered resistance to infection. Am J Clin Nutr 1998;68:447S-63S.

9. Lang C, Murgia C, Leong M, et al. Antiinflammatory effects of zinc and alterations in zinc transporter mRNA in mouse models of allergic inflammation. Am J Physiol Lung Cell Mol Physiol 2007;292: L577-84.

10. Truong-Tran AQ, Carter J, Ruffin R, et al. New insights into the role of zinc in the respiratory epithelium. Immunol Cell Biol 2001;79:170-7.

11. Zalewski PD, Truong-Tran AQ, Grosser D, et al. Zinc metabolism in airway epithelium and airway inflammation: basic mechanisms and clinical targets. A review. Pharmacol Ther 2005;105:127-49.

12. Jayaram L, Chunilal S, Pickering S, et al. Sputum zinc concentration and clinical outcome in older asthmatics. Respirology 2011;16:459-66.

13. Kadrabova J, Mad'aric A, Podivinsky F, et al. Plasma zinc, copper and copper/zinc ratio in intrinsic asthma. $\mathrm{J}$ Trace Elem Med Biol 1996;10:50-3.

14. Nurmatov U, Devereux G, Sheikh A. Nutrients and foods for the primary prevention of asthma and allergy: systematic review and meta-analysis. J Allergy Clin Immunol 2011;127:724-33 e1-30.

15. Razi CH, Akelma AZ, Akin 0, et al. Hair zinc and selenium levels in children with recurrent wheezing. Pediatr Pulmonol 2012;47:1185-91.

16. Schwartz J, Weiss ST. Dietary factors and their relation to respiratory symptoms. The second national health and nutrition examination survey. Am J Epidemiol 1990;132:67-76.

17. Khanbabaee G, Omidian A, Imanzadeh F, et al. Serum level of zinc in asthmatic patients: a case-control study. Allergol Immunopathol (Madr) 2014;42:19-21.

18. Biltagi MA, Baset AA, Bassiouny M, et al. Omega-3 fatty acids, vitamin $\mathrm{C}$ and $\mathrm{Zn}$ supplementation in asthmatic children: a randomized self-controlled study. Acta Paediatr 2009;98:737-42.

19. Ghaffari J, Khalilian A, Salehifar E, et al. Effect of zinc supplementation in children with asthma: a randomized, placebo-con- 
trolled trial in northern Islamic Republic of Iran. East Mediterr Health J 2014;20:391-6.

20. Pouramjad S, Egtesadi S, Moosavi S, et al. Study of zinc serum concentration and effect of zinc supplementation on lung function in asthmatic patients. J Iran Univ Med Sci 2009;15:55-61.

21. Cronin J, Kennedy U, McCoy S, et al. Single dose oral dexamethasone versus multi-dose prednisolone in the treatment of acute exacerbations of asthma in children who attend the emergency department: study protocol for a randomized controlled trial. Trials 2012;13:141.

22. Ducharme FM, Chalut D, Plotnick L, et al. The pediatric respiratory assessment measure: a valid clinical score for assessing acute asthma severity from toddlers to teenagers. J Pediatr 2008;152:476-80, 480.e1.

23. Hotz C, Peerson JM, Brown KH. Suggested lower cutoffs of serum zinc concentrations for assessing zinc status: reanalysis of the second national health and nutrition examination survey data (1976-1980). Am J Clin Nutr 2003;78:756-64.

24. el-Kholy MS, Gas Allah MA, el-Shimi S, et al. Zinc and copper status in children with bronchial asthma and atopic dermatitis. J Egypt Public Health Assoc 1990;65:657-68.

25. Brown KH, Rivera JA, Bhutta Z, et al. International Zinc Nutrition Consultative Group (IZiNCG) technical document \#1. Assessment of the risk of zinc deficiency in populations and options for its control. Food Nutr Bull 2004;25:S99-203.
26. Morgan CI, Ledford JR, Zhou P, et al. Zinc supplementation alters airway inflammation and airway hyperresponsiveness to a common allergen. J Inflamm (Lond) 2011;8:36.

27. Saper RB, Rash R. Zinc: an essential micronutrient. Am Fam Physician 2009;79:768-72.

28. Ortiz-Alvarez 0, Mikrogianakis A. Managing the paediatric patient with an acute asthma exacerbation. Paediatr Child Health 2012;17:251-62.

29. Murgia C, Grosser D, Truong-Tran $A Q$, et al. Apical localization of zinc transporter ZnT4 in human airway epithelial cells and its loss in a murine model of allergic airway inflammation. Nutrients 2011;3:91028. 